\title{
Nontrivial solutions for a fractional differential equation with nonlocal Riemann-Stieltjes boundary conditions
}

\author{
Xinguang Zhanga,c,*, Lishan Liub,c, Yonghong Wuc, Yujun Cuid \\ a School of Mathematical and Informational Sciences, Yantai University, Yantai 264005, Shandong, China. \\ ${ }^{b}$ School of Mathematical Sciences, Qufu Normal University, Qufu 273165, Shandong, China. \\ ${ }^{c}$ Department of Mathematics and Statistics, Curtin University of Technology, Perth, WA 6845, Australia. \\ ${ }^{d}$ Department of Mathematics, Shandong University of Science and Technology, Qingdao, 266590, Shandong, China. \\ Communicated by X.-J. Yang
}

\begin{abstract}
The model of fractional differential equation arises from various fields of physics, engineering, and applied mathematics. In this paper, we focus on the existence and uniqueness of nontrivial solutions for a abstract model of fractional differential equation with nonlocal Riemann-Stieltjes boundary conditions. Under certain suitable growth conditions, we establish some sufficient conditions for the existence and uniqueness of nontrivial solutions based on Leray-Schauder nonlinear alternative and Schauder fixed point theorem. (C)2017 All rights reserved.
\end{abstract}

Keywords: Fractional differential equation, nontrivial solution, nonlocal Riemann-Stieltjes boundary conditions, fixed point theorem.

2010 MSC: 34B15, 34B18.

\section{Introduction}

In this paper, we focus on the existence and uniqueness of nontrivial solutions for the following nonlocal fractional differential equation modelling fluid mechanics in a porous medium

$$
\left\{\begin{array}{l}
-\mathscr{D}_{\mathbf{t}}{ }^{\alpha} z(\mathrm{t})=\mathrm{f}\left(\mathrm{t}, z(\mathrm{t}), \mathscr{D}_{\mathbf{t}}^{\gamma} z(\mathrm{t})\right), \quad 0<\mathrm{t}<1, \\
\mathscr{D}_{\mathbf{t}}^{\gamma} z(0)=\mathscr{D}_{\mathbf{t}}{ }^{\gamma+1} z(0)=0, \mathscr{D}_{\mathbf{t}}{ }^{\mu} z(1)=\int_{0}^{1} \mathscr{D}_{\mathbf{t}}{ }^{\mu} z(s) \mathrm{d} A(s),
\end{array}\right.
$$

where $2<\alpha \leqslant 3$, and $0<\gamma \leqslant \alpha-2, \gamma \leqslant \mu<\alpha-1, \mathscr{D}_{\mathbf{t}}{ }^{\alpha}$ is the standard Riemann-Liouville derivative, $\int_{0}^{1} \mathscr{D}_{\mathbf{t}}^{\mu} \times(\mathrm{s}) \mathrm{d} A(\mathrm{~s})$ is a linear functional given by Riemann-Stieltjes integrals, $A$ is a function of bounded variation and $d A$ is allowed to be a changing-sign measure, and $f:[0,1] \times \mathbb{R} \rightarrow \mathbb{R}$ is continuous.

The model of fractional differential equation has great importance in describing various phenomena of fluid mechanics, biology, control theory of dynamical systems, viscoelasticity, physics and engineering.

\footnotetext{
*Corresponding author

Email address: zxg123242@163.com (Xinguang Zhang)
}

doi:10.22436/jnsa.010.11.39 
Recently, Laskin [9-12] initiated to use the fractional Schrödinger equation to model quantum mechanics, where the fractional Schrödinger equation includes a spatial derivative of fractional order. Mckee and Cuminato [15] introduced a novel variant of product integration to deal with multiple integrable singularities and showed numerically to exist an unexpected convergence rates through a discrete Abel's equation. In [31], Zhang et al. established analytic solutions for a fractional model of turbulent flow in a porous medium by using the fixed point theorem of the mixed monotone operator. By establishing a variational structure and using the critical point theory, Zhang et al. [32] investigated the existence of multiple solutions for a class of fractional advection-dispersion equations arising from a symmetric transition of the mass flux. In heat transfer, Yang et al. [23] introduced some new general fractional derivatives involving the kernels of the extended Mittag-Leffler type function to analyze the complex phenomena for the anomalous diffusion. Recently, some further study on fractional derivatives of constant and without singular kernel in heat transfer has also been carried out in work of Yang [22] and Yang et al. [24]. For an extensive collection of work about application of fractional order differential equation, we refer the reader to Diethelm et al. [5] in viscoplasticity, [3] in fractal mediums, Glockle and Nonnenmacher [6] in protein dynamics, Mainardi [14] in continuum and statistical mechanics, Azar [1] in control and synchronization of chaotic systems and so on.

In mathematical frame, many works focused on analytic solutions and numerical solutions for the fractional model, see our works [2, 13, 18-20, 25-30,33-36]. Recently, by using cone theoretic techniques, Goodrich [7] established a general existence theorem for the following fractional boundary value problem

$$
\left\{\begin{array}{c}
-\mathscr{D}_{\mathbf{t}}{ }^{\alpha} z(\mathrm{t})=\mathrm{f}(\mathrm{t}, z(\mathrm{t})), \quad 0<\mathrm{t}<1, \mathrm{n}-1<\alpha \leqslant \mathrm{n}, \mathrm{n}>3, \\
z^{(i)}(0)=0,0 \leqslant i \leqslant n-2, \mathscr{D}_{\mathbf{t}}{ }^{\alpha} z(1)=0, \quad 1 \leqslant \alpha \leqslant n-2,
\end{array}\right.
$$

when $f(t, z)$ satisfies some growth conditions. And then, Jiang et al. [8] considered a fractional differential equation

$$
\mathscr{D}_{\mathbf{t}}{ }^{\alpha} z(t)=f(t, z(t)), \quad t \in(0,1)
$$

subject to multi-point boundary conditions

$$
z(0)=0, \quad \mathscr{D}_{\mathbf{t}}{ }^{\alpha} z(1)=\sum_{i=1}^{m-2} a_{i} \mathscr{D}_{\mathbf{t}}{ }^{\alpha} z\left(\xi_{\mathbf{i}}\right),
$$

where $1<\alpha \leqslant 2,0<\beta<1,0<\xi_{1}<\xi_{i}<\ldots<\xi_{m-2}<1, a_{i} \in[0,+\infty)$ with $\sum_{i=1}^{m-2} a_{i} \xi_{i}^{\alpha-\beta-1}<1$, by using the fixed point index theory and Krein-Rutman theorem, the authors established the existence of positive solutions for the above fractional order multi-point boundary value problems provided that the nonlinear function $f:[0,1] \times \mathbb{R}^{+} \rightarrow \mathbb{R}^{+}$satisfies Carathéodory condition and certain growth conditions.

Notice that the nonlinearity of the above work does not contain fractional derivatives of unknown functions and boundary condition is not Riemann-Stieltjes integrals types. Thus the aim of this paper is to establish the existence and uniqueness of nontrivial solutions for (1.1) when nonlinear term $f$ and the boundary conditions all contain fractional derivatives of unknown functions and boundary condition is nonlocal Riemann-Stieltjes integrals. To the best of our knowledge, the fractional order flow model with Riemann-Stieltjes integral conditions has been seldom considered when $f$ and boundary conditions all contain fractional derivatives of unknown functions. In this work, by introducing a fractional integral operator, we reduce the higher order (1.1) to a lower order integro-differential equation, and then by means of Leray-Schauder nonlinear alternative and Schauder fixed point theorem, we obtain some new results of existence and uniqueness of nontrivial solutions for (1.1). Some examples are also given to illustrate the application of the main results.

\section{Basic definitions and preliminaries}

The main results of this paper is restricted in the sense of Riemann-Liouville fractional calculus, and for the convenience of readers, here we give the related definitions and properties which are used in the rest of this paper. 
Definition 2.1 ([16, 17]). The Riemann-Liouville fractional integral of order $\alpha>0$ of a function $z$ : $(0,+\infty) \rightarrow \mathbb{R}$ is given by

$$
\mathrm{I}^{\alpha} z(\mathrm{t})=\frac{1}{\Gamma(\alpha)} \int_{0}^{\mathrm{t}}(\mathrm{t}-\mathrm{s})^{\alpha-1} z(\mathrm{~s}) \mathrm{d} s
$$

provided that the right-hand side is pointwise defined on $(0,+\infty)$.

Definition $2.2([16,17])$. The Riemann-Liouville fractional derivative of order $\alpha>0$ of a continuous function $z:(0,+\infty) \rightarrow \mathbb{R}$ is given by

$$
\mathscr{D}_{t}^{\alpha} z(t)=\frac{1}{\Gamma(n-\alpha)}\left(\frac{d}{d t}\right)^{(n)} \int_{0}^{t}(t-s)^{n-\alpha-1} z(s) d s,
$$

where $n=[\alpha]+1,[\alpha]$ denotes the integer part of the number $\alpha$, provided that the right-hand side is pointwise defined on $(0,+\infty)$.

Definition 2.3. A function $z$ is called a solution of (1.1) if $z \in C[0,1]$ and satisfies (1.1). In addition, $z$ is said to be a nontrivial solution of (1.1) if $z$ is solution of (1.1) and $z \not \equiv 0$ for $t \in(0,1)$.

Proposition $2.4([16,17])$.

1. If $z, y:(0,+\infty) \rightarrow \mathbb{R}$ and $\alpha>0$, then

$$
\mathscr{D}_{\mathbf{t}}^{\alpha}(z(\mathrm{t})+\mathrm{y}(\mathrm{t}))=\mathscr{D}_{\mathbf{t}}^{\alpha} z(\mathrm{t})+\mathscr{D}_{\mathbf{t}}{ }^{\alpha} \mathrm{y}(\mathrm{t})
$$

2. If $z \in \mathrm{L}^{1}(0,1), \alpha>\beta>0$, then

$$
\mathrm{I}^{\alpha} \mathrm{I}^{\beta} z(\mathrm{t})=\mathrm{I}^{\alpha+\beta} z(\mathrm{t}), \quad \mathscr{D}_{\mathbf{t}}^{\beta} \mathrm{I}^{\alpha} z(\mathrm{t})=\mathrm{I}^{\alpha-\beta} z(\mathrm{t}), \quad \mathscr{D}_{\mathbf{t}}{ }^{\beta} \mathrm{I}^{\beta} z(\mathrm{t})=z(\mathrm{t}) .
$$

3. If $\alpha>0, \beta>0$, then

$$
\mathscr{D}_{\mathbf{t}}{ }^{\alpha} t^{\beta-1}=\frac{\Gamma(\beta)}{\Gamma(\beta-\alpha)} t^{\beta-\alpha-1}
$$

4. Let $\alpha>0$, and $f(x)$ is integrable, then

$$
I^{\alpha} \mathscr{D}_{t}^{\alpha} f(t)=f(t)+c_{1} t^{\alpha-1}+c_{2} t^{\alpha-2}+\cdots+c_{n} t^{\alpha-n},
$$

where $c_{i} \in \mathbb{R}(i=1,2, \cdots, n), n$ is the smallest integer greater than or equal to $\alpha$.

Lemma 2.5. Assume $2<\alpha-\gamma \leqslant 3$, then (1.1) is equivalent to the following integro-differential equation

$$
\left\{\begin{array}{l}
-\mathscr{D}_{t}{ }^{\alpha-\gamma} y(t)=f\left(t, I^{\gamma} y(t), y(t)\right) \\
y(0)=y^{\prime}(0)=0, \quad \mathscr{D}^{\mu-\gamma} y(1)=\int_{0}^{1} \mathscr{D}^{\mu-\gamma} y(s) d A(s) .
\end{array}\right.
$$

Proof. In fact, let $z(t)=\mathrm{I}^{\gamma} \mathrm{y}(\mathrm{t})$ and $\mathrm{y} \in \mathrm{C}[0,1]$, it follows from Proposition 2.4 that

$$
\begin{aligned}
\mathscr{D}_{\mathbf{t}}{ }^{\alpha} z(t) & =\frac{\mathrm{d}^{\mathrm{n}}}{\mathrm{dt}^{n}} \mathrm{I}^{\mathrm{n}-\alpha} z(\mathrm{t})=\frac{\mathrm{d}^{\mathrm{n}}}{\mathrm{dt}^{\mathrm{n}}} \mathrm{I}^{\mathrm{n}-\alpha} \mathrm{I}^{\gamma} \mathrm{y}(\mathrm{t})=\frac{\mathrm{d}^{\mathrm{n}}}{\mathrm{dt}^{\mathrm{n}}} \mathrm{I}^{\mathrm{n}-\alpha+\gamma} \mathrm{y}(\mathrm{t})=\mathscr{D}_{\mathbf{t}}{ }^{\alpha-\gamma} y(\mathrm{t}), \\
\mathscr{D}_{\mathbf{t}}{ }^{\gamma} z(\mathrm{t}) & =\mathscr{D}_{\mathbf{t}}{ }^{\gamma} \mathrm{I}^{\gamma} \mathrm{y}(\mathrm{t})=\mathrm{y}(\mathrm{t}), \\
\mathscr{D}_{\mathbf{t}}{ }^{\gamma+1} z(\mathrm{t}) & =\mathscr{D}_{\mathbf{t}}{ }^{\gamma+1} \mathrm{I}^{\gamma} \mathrm{y}(\mathrm{t})=\mathrm{y}^{\prime}(\mathrm{t}) .
\end{aligned}
$$

Thus by (2.2), one has $-\mathscr{D}_{t}{ }^{\alpha-\gamma} y(t)=f\left(t, I^{\gamma} y(t), y(t)\right)$, and

$$
\mathscr{D}_{\mathbf{t}}^{\gamma} z(0)=y(0)=0, \mathscr{D}_{\mathbf{t}}^{\gamma+1} z(0)=y^{\prime}(0)=0, \mathscr{D}_{\mathbf{t}}^{\mu-\gamma} \mathrm{y}(1)=\int_{0}^{1} \mathscr{D}_{\mathbf{t}}^{\mu-\gamma} \mathrm{y}(\mathrm{s}) \mathrm{dA}(\mathrm{s}),
$$


which implies that (1.1) is transformed into the integro-differential equation (2.1).

On the other hand, if $y \in C[0,1]$ solves the integro-differential equation (2.1), then $y$ satisfies the equation (2.1), thus by (2.2), we have

$$
-\mathscr{D}_{\mathbf{t}}^{\alpha} z(t)=-\mathscr{D}_{t}^{\alpha-\gamma} y(t)=f\left(t, I^{\gamma} y(t), y(t)\right)=f\left(t, z(t), \mathscr{D}_{t}^{\gamma} z(t)\right),
$$

and

$$
\mathscr{D}_{\mathbf{t}}^{\gamma} z(0)=y(0)=0, \mathscr{D}_{\mathbf{t}}^{\gamma} z(0)=y^{\prime}(0)=0, \mathscr{D}_{\mathbf{t}}{ }^{\mu} z(1)=\int_{0}^{1} \mathscr{D}_{\mathbf{t}}{ }^{\mu} z(s) \mathrm{d} A(s) .
$$

Consequently, (1.1) is equivalent to the integro-differential equation (2.1).

Lemma 2.6. Given $y \in \mathrm{L}^{1}(0,1)$, then the boundary value problem

$$
\left\{\begin{array}{c}
-\mathscr{D}_{\mathbf{t}}{ }^{\alpha-\gamma} \mathrm{y}(\mathrm{t})=\mathrm{h}(\mathrm{t}), 0<\mathrm{t}<1 \\
\mathrm{y}(0)=\mathrm{y}^{\prime}(0)=0, \mathscr{D}_{\mathbf{t}}^{\mu-\gamma} \mathrm{y}(1)=0
\end{array}\right.
$$

has the unique solution

$$
y(t)=\int_{0}^{1} G(t, s) h(s) d s
$$

where $\mathrm{G}(\mathrm{t}, \mathrm{s})$ is the Green function of the boundary value problem (2.3) and is given by

$$
G(t, s)= \begin{cases}\frac{t^{\alpha-\gamma-1}(1-s)^{\alpha-\mu-1}-(t-s)^{\alpha-\gamma-1}}{\Gamma(\alpha-\gamma)}, & 0 \leqslant s \leqslant t \leqslant 1 \\ \frac{t^{\alpha-\gamma-1}(1-s)^{\alpha-\mu-1}}{\Gamma(\alpha-\gamma)}, & 0 \leqslant t \leqslant s \leqslant 1\end{cases}
$$

Proof. From Proposition 2.4, (2.1) can be reduced to the following equivalent integral equation

$$
y(t)=-I^{\alpha-\gamma} h(t)+c_{1} t^{\alpha-\gamma-1}+c_{2} t^{\alpha-\gamma-2}+c_{3} t^{\alpha-\gamma-3}, c_{1}, c_{2}, c_{3} \in \mathbb{R} .
$$

Since $y(0)=y^{\prime}(0)=0$, it follows from (2.5) that $c_{2}=c_{2}=0$. Consequently the general solution of (2.1) satisfies

$$
y(t)=-I^{\alpha-\gamma} h(t)+c_{1} t^{\alpha-\gamma-1}=-\int_{0}^{t} \frac{(t-s)^{\alpha-\gamma-1}}{\Gamma(\alpha-\gamma)} h(s) d s+c_{1} t^{\alpha-\gamma-1} .
$$

It follows from Proposition 2.4 and (2.6) that

$$
\begin{aligned}
\mathscr{D}^{\mu-\gamma} y(t) & =-\mathscr{D}^{\mu-\gamma} I^{\alpha-\gamma} h(t)+c_{1} \mathscr{D}^{\mu-\gamma} t^{\alpha-\gamma-1}=-I^{\alpha-\mu} h(t)+c_{1} \frac{\Gamma(\alpha-\gamma)}{\Gamma(\alpha-\mu)} t^{\alpha-\mu-1} \\
& =-\int_{0}^{t} \frac{(t-s)^{\alpha-\mu-1}}{\Gamma(\alpha-\mu)} h(s) d s+c_{1} \frac{\Gamma(\alpha-\gamma)}{\Gamma(\alpha-\mu)} t^{\alpha-\mu-1} .
\end{aligned}
$$

So, from (2.7) and $\mathscr{D}_{\mathbf{t}}^{\mu-\gamma} \mathrm{y}(1)=0$, we have

$$
\mathscr{D}^{\mu-\gamma} \mathrm{y}(1)=-\int_{0}^{1} \frac{(1-s)^{\alpha-\mu-1}}{\Gamma(\alpha-\mu)} \mathrm{h}(\mathrm{s}) \mathrm{d} s+\mathrm{c}_{1} \frac{\Gamma(\alpha-\gamma)}{\Gamma(\alpha-\mu)}=0,
$$

i.e.,

$$
c_{1}=\int_{0}^{1} \frac{(1-s)^{\alpha-\mu-1}}{\Gamma(\alpha-\gamma)} h(s) d s .
$$

So, by (2.6), the unique solution of problem (2.1) is

$$
y(t)=-\int_{0}^{t} \frac{(t-s)^{\alpha-\gamma-1}}{\Gamma(\alpha-\gamma)} h(s) d s+\int_{0}^{1} \frac{t^{\alpha-\gamma-1}(1-s)^{\alpha-\mu-1}}{\Gamma(\alpha-\gamma)} h(s) d s=\int_{0}^{1} G(t, s) h(s) d s .
$$

The proof is completed. 
On the other hand, from Proposition 2.4, we know the unique solution of the following boundary value problem

$$
\left\{\begin{array}{l}
\mathscr{D}^{\alpha-\gamma} \mathrm{y}(\mathrm{t})=0,0<\mathrm{t}<1 \\
\mathrm{y}(0)=\mathrm{y}^{\prime}(0)=0, \mathscr{D}_{\mathbf{t}}^{\mu-\gamma} \mathrm{y}(1)=1
\end{array}\right.
$$

is $\frac{\Gamma(\alpha-\mu)}{\Gamma(\alpha-\gamma)} \mathrm{t}^{\alpha-\gamma-1}$. Thus let

$$
\mathcal{C}=\int_{0}^{1} \frac{\Gamma(\alpha-\mu)}{\Gamma(\alpha-\gamma)} \mathrm{t}^{\alpha-\gamma-1} \mathrm{dA}(\mathrm{t}), \quad \mathcal{B}=\frac{\Gamma(\alpha-\mu)}{(1-\mathcal{C}) \Gamma(\alpha-\gamma)}
$$

and define

$$
\mathcal{G}_{A}(s)=\int_{0}^{1} G(t, s) d A(t) .
$$

According to the strategy of [21], we know the Green function of the integro-differential equation (2.1) is

$$
K(t, s)=B t^{\alpha-\gamma-1} \mathcal{G}_{A}(s)+G(t, s) .
$$

And then, we have the following lemma.

Lemma 2.7. Let $\mathrm{h} \in \mathrm{L}^{1}[0,1]$, if $2<\alpha \leqslant 3$, and $0<\gamma \leqslant \alpha-2, \gamma \leqslant \mu<\alpha$. Then the fractional boundary value problem

has unique solution

$$
\left\{\begin{array}{l}
-\mathscr{D}_{\mathbf{t}}{ }^{\alpha-\gamma} \mathrm{y}(\mathrm{t})=\mathrm{h}(\mathrm{t}), \\
\mathrm{y}(0)=\mathrm{y}^{\prime}(0)=0, \quad \mathscr{D}_{\mathrm{t}}^{\mu-\gamma} \mathrm{y}(1)=\int_{0}^{1} \mathscr{D}_{\mathbf{t}}^{\mu-\gamma} \mathrm{y}(\mathrm{s}) \mathrm{dA}(\mathrm{s}) .
\end{array}\right.
$$

$$
y(t)=\int_{0}^{1} K(t, s) h(s) d s
$$

where $\mathrm{K}(\mathrm{t}, \mathrm{s})$ is defined by (2.8).

Lemma 2.8. The Green function defined by (2.8) satisfies:

$$
|\mathrm{K}(\mathrm{t}, \mathrm{s})| \leqslant \mathcal{H}(\mathrm{s}) \leqslant \frac{(1-\mathrm{s})^{\alpha-\mu-1}}{\Gamma(\alpha-\gamma)}+\mathcal{E}
$$

where

$$
\mathcal{H}(s)=\frac{(1-s)^{\alpha-\mu-1}}{\Gamma(\alpha-\gamma)}+\mathcal{B}\left|\mathcal{G}_{A}(s)\right| .
$$

Proof. By (2.4) and (2.8), we have

$$
|K(t, s)|=\mathcal{B} t^{\alpha-\gamma-1}\left|\mathcal{G}_{A}(s)\right|+G(t, s) \leqslant \frac{t^{\alpha-\gamma-1}(1-s)^{\alpha-\mu-1}}{\Gamma(\alpha-\gamma)}+\mathcal{B}\left|\mathcal{G}_{A}(s)\right|=\mathcal{H}(s) \leqslant \frac{(1-s)^{\alpha-\mu-1}}{\Gamma(\alpha-\gamma)}+\mathcal{E},
$$

where $\mathcal{E}=\mathcal{B}\left\|\mathcal{G}_{\mathrm{A}}(\mathrm{s})\right\|$.

Define an operator $\mathrm{T}: \mathrm{C}[0,1] \rightarrow \mathrm{C}[0,1]$ by

$$
(T y)(t)=\int_{0}^{1} K(t, s) f\left(s, I^{\gamma} y(s), y(s)\right) d s,
$$

By Lemmas 2.7 and 2.5, the fixed point $y$ of operator T is the solution of $(2.1)$, and then $z(t)=I^{\gamma} y(t)$ is a solution of (1.1).

Lemma 2.9. The operator $\mathrm{T}: \mathrm{C}[0,1] \rightarrow \mathrm{C}[0,1]$ is completely continuous.

Proof. Noticing that $f:[0,1] \times \mathbb{R}^{2} \rightarrow \mathbb{R}$ is continuous and $K(t, s)$ is uniformly continuous in $[0,1] \times[0,1]$, by the Ascoli-Arzelà theorem, $\mathrm{T}: \mathrm{C}[0,1] \rightarrow \mathrm{C}[0,1]$ is completely continuous. 


\section{Main results}

Before begin to state our main result, we first introduce the following lemma.

Lemma 3.1 ([4, Leray-Schauder alternative]). Let $\mathrm{E}$ be a real Banach space, and $\Omega$ be a bounded open subset of $\mathrm{E}$, where $\theta \in \Omega, \mathrm{T}: \bar{\Omega} \rightarrow \mathrm{E}$ is a completely continuous operator. Then, either there exists $w \in \partial \Omega, \lambda>1$ such that $\mathrm{T} w=\lambda w$, or there exists a fixed point $w^{*} \in \bar{\Omega}$.

Theorem 3.2. Assume $\mathrm{f}(\mathrm{t}, 0,0) \not \equiv 0$ for any $\mathrm{t} \in[0,1]$, and there exist three nonnegative functions $\mathrm{p}, \mathrm{q}, \mathrm{r} \in \mathrm{L}^{1}[0,1]$ such that

$$
\left|\mathbf{f}\left(\mathrm{t}, z_{1}, z_{2}\right)\right| \leqslant \mathrm{p}(\mathrm{t})\left|z_{1}\right|+\mathrm{q}(\mathrm{t})\left|z_{2}\right|+\mathrm{r}(\mathrm{t}), \text { a.e. }\left(\mathrm{t}, z_{1}, z_{2}\right) \in[0,1] \times \mathbb{R}^{2},
$$

Then (1.1) has at least one nontrivial solution provided that

$$
\int_{0}^{1} \mathcal{H}(s)[p(s)+q(s)] d s<\left(1+\frac{1}{\Gamma(\gamma+1)}\right)^{-1}
$$

where $\mathcal{H}(s)$ is given by (2.9).

Proof. Firstly, it follows from $f(t, 0, \ldots, 0) \not \equiv 0$ that there exists $\left[t_{1}, t_{2}\right] \in[0,1]$ such that

$$
\min _{t \in\left[t_{1}, t_{2}\right]}|f(t, 0,0)|>0 .
$$

Since

$$
\left|\mathbf{f}\left(\mathbf{t}, z_{1}, z_{2}\right)\right| \leqslant p(t)\left|z_{1}\right|+q(t)\left|z_{2}\right|+r(t) \text { a.e. }\left(t, z_{1}, z_{2}\right) \in[0,1] \times \mathbb{R}^{2},
$$

one has

$$
r(t) \geqslant|f(t, 0,0)|, \text { a.e. } t \in[0,1] .
$$

Consequently, by (3.3), (3.4), we get

$$
\int_{0}^{1} \mathcal{H}(s) r(s) d s \geqslant \int_{t_{2}}^{t_{1}} \mathcal{H}(s) f(s, 0,0) d s>0 .
$$

On the other hand, from (3.2), we know

$$
\left(1+\frac{1}{\Gamma(\gamma+1)}\right) \int_{0}^{1} \mathcal{H}(\mathrm{s})[\mathrm{p}(\mathrm{s})+\mathrm{q}(\mathrm{s})] \mathrm{d} \mathrm{s}<1 .
$$

Thus take a constant

$$
\sigma=\frac{\int_{0}^{1} \mathcal{H}(\mathrm{s}) \mathrm{r}(\mathrm{s}) \mathrm{ds}}{1-\left(1+\frac{1}{\Gamma(\gamma+1)}\right) \int_{0}^{1} \mathcal{H}(\mathrm{s})[\mathrm{p}(\mathrm{s})+\mathrm{q}(\mathrm{s})] \mathrm{ds}},
$$

then $\sigma>0$.

Now let $\Omega_{\sigma}=\{y \in C[0,1]:\|x\|<\sigma\}$. Suppose that there exist $y_{0} \in \partial \Omega_{\sigma}, \lambda>1$ such that $T y_{0}=\lambda y_{0}$. Then

$$
\begin{aligned}
\lambda \sigma=\lambda\left\|y_{0}\right\|=\left\|T y_{0}\right\| & =\max _{t \in[0,1]}\left|\int_{0}^{1} K(t, s) f\left(s, I^{\gamma} y_{0}(s), y_{0}(s)\right) d s\right| \\
& \leqslant \int_{0}^{1} \mathcal{H}(s)\left|f\left(s, I^{\gamma} y_{0}(s), y_{0}(s)\right)\right| d s .
\end{aligned}
$$

Since

$$
\left|I^{\gamma} \mathrm{y}_{0}(\mathrm{t})\right|=\left|\int_{0}^{\mathrm{t}} \frac{(\mathrm{t}-\mathrm{s})^{\gamma-1} \mathrm{y}_{0}(\mathrm{~s})}{\Gamma(\gamma)} \mathrm{ds}\right| \leqslant \frac{\left\|\mathrm{y}_{0}\right\|}{\Gamma(\gamma+1)}
$$


by hypothesis (3.1), one has

$$
\begin{aligned}
\left|f\left(s, I^{\gamma} y_{0}(s), y_{0}(s)\right)\right| \leqslant p(s)\left|I^{\gamma} y_{0}(s)\right|+q(s)\left|y_{0}(s)\right|+r(s) & \leqslant \frac{\left\|y_{0}\right\|}{\Gamma(\gamma+1)} p(s)+q(s)\left\|y_{0}\right\|+r(s) \\
& \leqslant\left(1+\frac{1}{\Gamma(\gamma+1)}\right)[p(s)+q(s)]\left\|y_{0}\right\|+r(s) .
\end{aligned}
$$

It follows from (3.5) and (3.6) that

$$
\begin{aligned}
\lambda \sigma & \leqslant\left(1+\frac{1}{\Gamma(\gamma+1)}\right) \int_{0}^{1} \mathcal{H}(\mathrm{s})[\mathrm{p}(\mathrm{s})+\mathrm{q}(\mathrm{s})] \mathrm{d} s\left\|\mathrm{y}_{0}\right\|+\int_{0}^{1} \mathcal{H}(\mathrm{s}) \mathrm{r}(\mathrm{s}) \mathrm{d} s \\
& =\sigma\left(1+\frac{1}{\Gamma(\gamma+1)}\right) \int_{0}^{1} \mathcal{H}(\mathrm{s})[\mathrm{p}(\mathrm{s})+\mathrm{q}(\mathrm{s})] \mathrm{d} s+\int_{0}^{1} \mathcal{H}(\mathrm{s}) \mathrm{r}(\mathrm{s}) \mathrm{d} s .
\end{aligned}
$$

That is,

$$
\lambda \leqslant\left(1+\frac{1}{\Gamma(\gamma+1)}\right) \int_{0}^{1} \mathcal{H}(s)[p(s)+q(s)] d s+\frac{\int_{0}^{1} \mathcal{H}(s) r(s) d s}{\sigma}=1,
$$

which contradicts with $\lambda>1$. By Lemma 3.1, T has a fixed point $y^{*} \in \bar{\Omega}$, since $f(t, 0,0) \not \equiv 0$, by Lemma 2.7, the solution $\mathrm{y}^{*}$ of (2.1) is nontrivial, furthermore, (1.1) has at least a nontrivial solution $z^{*}=\mathrm{I}^{\gamma} \mathrm{y}^{*}$. This completes the proof.

Corollary 3.3. Assume that (3.1) holds and $\mathrm{f}(\mathrm{t}, 0,0) \not \equiv 0$ for any $\mathrm{t} \in[0,1]$. Then (1.1) has at least one nontrivial solution if one of the following conditions is satisfied.

(1) There exists a constant $p>1$ such that

$$
\int_{0}^{1}[p(s)+q(s)]^{p} d s<\left[\frac{1}{\Gamma(\alpha-\gamma)}\left(\frac{p(\alpha-\mu-1)}{p-1}+1\right)^{\frac{p-1}{p}}+\varepsilon\right]^{-p}\left(1+\frac{1}{\Gamma(\gamma+1)}\right)^{-p} .
$$

(2) There exists a constant $\lambda>-1$ such that

$$
\mathrm{p}(\mathrm{s})+\mathrm{q}(\mathrm{s})<\left[\frac{\Gamma(\alpha-\mu) \Gamma(\lambda+1)}{\Gamma(\alpha+\lambda-\mu-1) \Gamma(\alpha-\gamma)}+\frac{\mathcal{E}}{\lambda+1}\right]^{-1}\left(1+\frac{1}{\Gamma(\gamma+1)}\right)^{-1} \mathrm{~s}^{\lambda} .
$$

(3) There exists a constant $\lambda>-1$ such that

$$
p(s)+q(s)<\frac{(\lambda+1)(\alpha+\lambda-\mu) \Gamma(\alpha-\gamma)}{\lambda+\varepsilon+1}\left(1+\frac{1}{\Gamma(\gamma+1)}\right)^{-1}(1-s)^{\lambda} .
$$

(4) $p_{i}(s)(i=1,2, \cdots, n)$ satisfy

$$
p(s)+q(s)<(\alpha-\mu)\left(M+\sum_{i=0}^{n-2} \frac{M}{\Gamma\left(\gamma-\mu_{i}\right)}\right)^{-1} .
$$

Proof. Take

$$
\mathrm{R}=\int_{0}^{1} \mathcal{H}(\mathrm{s})[\mathrm{p}(\mathrm{s})+\mathrm{q}(\mathrm{s})] \mathrm{d} \mathrm{s} .
$$

According to the proof of Theorem 3.2, we only need to show

$$
\mathrm{R}<\left(1+\frac{1}{\Gamma(\gamma+1)}\right)^{-1}
$$


(1) If (3.7) is valid, let $\frac{1}{p}+\frac{1}{q}=1$, and by means of Hölder inequality, we have

$$
\begin{aligned}
\mathrm{R} & \leqslant \frac{1}{\Gamma(\alpha-\gamma)}\left(\int_{0}^{1}[\mathrm{p}(\mathrm{s})+\mathrm{q}(\mathrm{s})]^{\mathrm{p}} \mathrm{ds}\right)^{\frac{1}{\mathrm{p}}}\left(\int_{0}^{1}(1-\mathrm{s})^{\mathrm{q}(\alpha-\mu-1)} \mathrm{ds}\right)^{\frac{1}{\mathrm{q}}}+\mathcal{E}\left(\int_{0}^{1}[\mathrm{p}(\mathrm{s})+\mathrm{q}(\mathrm{s})]^{\mathrm{p}} \mathrm{ds}\right)^{\frac{1}{\mathrm{p}}} \\
& =\frac{1}{\Gamma(\alpha-\gamma)}\left[\frac{\mathrm{p}(\alpha-\mu-1)}{\mathrm{p}-1}+1\right]^{-\frac{\mathrm{p}-1}{\mathrm{p}}}\left(\int_{0}^{1}[\mathrm{p}(\mathrm{s})+\mathrm{q}(\mathrm{s})]^{\mathrm{p}} \mathrm{ds}\right)^{\frac{1}{\mathrm{p}}}+\mathcal{E}\left(\int_{0}^{1}[\mathrm{p}(\mathrm{s})+\mathrm{q}(\mathrm{s})]^{\mathrm{p}} \mathrm{ds}\right)^{\frac{1}{\mathrm{p}}} \\
& <\left(1+\frac{1}{\Gamma(\gamma+1)}\right)^{-1}
\end{aligned}
$$

(2) In this case, it follows from (3.8) that

$$
\begin{aligned}
\mathrm{R} & <\left[\frac{\Gamma(\alpha-\mu) \Gamma(\lambda+1)}{\Gamma(\alpha+\lambda-\mu-1) \Gamma(\alpha-\gamma)}+\frac{\mathcal{E}}{\lambda+1}\right]^{-1}\left(1+\frac{1}{\Gamma(\gamma+1)}\right)^{-1} \int_{0}^{1}\left[\frac{1}{\Gamma(\alpha-\gamma)}(1-s)^{\alpha-\mu-1}+\mathcal{E}\right] \mathrm{s}^{\lambda} \mathrm{d} s \\
& =\left(1+\frac{1}{\Gamma(\gamma+1)}\right)^{-1} .
\end{aligned}
$$

(3) If (3.9) holds, we get

$$
\begin{aligned}
R & <\frac{(\lambda+1)(\alpha+\lambda-\mu) \Gamma(\alpha-\gamma)}{\lambda+\mathcal{E}+1}\left(1+\frac{1}{\Gamma(\gamma+1)}\right)^{-1} \int_{0}^{1}\left[\frac{1}{\Gamma(\alpha-\gamma)}(1-s)^{\alpha-\mu-1}+\mathcal{E}\right](1-s)^{\lambda} \mathrm{d} s \\
& =\left(1+\frac{1}{\Gamma(\gamma+1)}\right)^{-1} .
\end{aligned}
$$

(4) In this case, from (3.10), we have

$$
\mathrm{R}<\frac{(\alpha-\mu) \Gamma(\alpha-\gamma)}{1+\mathcal{E}(\alpha-\mu) \Gamma(\alpha-\gamma)}\left(1+\frac{1}{\Gamma(\gamma+1)}\right)^{-1} \int_{0}^{1}\left[\frac{1}{\Gamma(\alpha-\gamma)}(1-s)^{\alpha-\mu-1}+\varepsilon\right] \mathrm{d} s=\left(1+\frac{1}{\Gamma(\gamma+1)}\right)^{-1} .
$$

The proof of Corollary 3.3 is completed.

Corollary 3.4. Suppose $\mathrm{f}(\mathrm{t}, 0,0) \not \equiv 0$ for any $\mathrm{t} \in[0,1]$ and

$$
0 \leqslant \limsup _{\left|z_{1}\right|+\left|z_{2}\right| \rightarrow+\infty} \max _{\mathfrak{t} \in[0,1]} \frac{\left|\mathbf{f}\left(\mathrm{t}, z_{1}, z_{2}\right)\right|}{\left|z_{1}\right|+\left|z_{2}\right|}<\frac{(\alpha-\mu) \Gamma(\alpha-\gamma)}{2+2 \mathcal{E}(\alpha-\mu) \Gamma(\alpha-\gamma)}\left(1+\frac{1}{\Gamma(\gamma+1)}\right)^{-1} .
$$

Then (1.1) has at least one nontrivial solution.

Proof. Take $\epsilon>0$ such that

$$
\frac{(\alpha-\mu) \Gamma(\alpha-\gamma)}{2+2 \mathcal{E}(\alpha-\mu) \Gamma(\alpha-\gamma)}\left(1+\frac{1}{\Gamma(\gamma+1)}\right)^{-1}-\epsilon>0,
$$

it follows from (3.11) that there exists a sufficiently large constant $R_{0}>0$ such that

$$
\left|f\left(t, z_{1}, z_{2}\right)\right| \leqslant\left(\frac{(\alpha-\mu) \Gamma(\alpha-\gamma)}{2+2 \mathcal{E}(\alpha-\mu) \Gamma(\alpha-\gamma)}\left(1+\frac{1}{\Gamma(\gamma+1)}\right)^{-1}-\epsilon\right)\left(\left|z_{1}\right|+\left|z_{2}\right|\right), \quad t \in[0,1],\left|z_{1}\right|+\left|z_{2}\right| \geqslant R_{0} .
$$

Let

$$
\hbar=\max _{\mathrm{t} \in[0,1],\left|z_{1}\right|+\left|z_{2}\right| \leqslant \mathrm{R}_{0}}\left|\mathbf{f}\left(\mathrm{t}, z_{1}, z_{2}\right)\right|,
$$


then for any $\left(t, z_{1}, z_{2}\right) \in[0,1] \times \mathbb{R}^{2}$, we have

$$
\left|f\left(t, z_{1}, z_{2}\right)\right| \leqslant \hbar+\left(\frac{(\alpha-\mu) \Gamma(\alpha-\gamma)}{2+2 \mathcal{E}(\alpha-\mu) \Gamma(\alpha-\gamma)}\left(1+\frac{1}{\Gamma(\gamma+1)}\right)^{-1}-\epsilon\right)\left(\left|z_{1}\right|+\left|z_{2}\right|\right) .
$$

Take

$$
p(s)+q(s)=2\left(\frac{(\alpha-\mu) \Gamma(\alpha-\gamma)}{2+2 \mathcal{E}(\alpha-\mu) \Gamma(\alpha-\gamma)}\left(1+\frac{1}{\Gamma(\gamma+1)}\right)^{-1}-\epsilon\right), r(s)=\hbar,
$$

then

$$
\mathrm{R}<\left(1+\frac{1}{\Gamma(\gamma+1)}\right)^{-1}
$$

In fact,

$$
\begin{aligned}
R & =\int_{0}^{1} \mathcal{H}(\mathrm{s})[\mathrm{p}(\mathrm{s})+\mathrm{q}(\mathrm{s})] \mathrm{d} s \\
& \leqslant 2\left(\frac{(\alpha-\mu) \Gamma(\alpha-\gamma)}{2+2 \mathcal{E}(\alpha-\mu) \Gamma(\alpha-\gamma)}\left(1+\frac{1}{\Gamma(\gamma+1)}\right)^{-1}-\epsilon\right) \int_{0}^{1}\left[\frac{1}{\Gamma(\alpha-\gamma)}(1-s)^{\alpha-\mu-1}+\mathcal{E}\right] \mathrm{d} s \\
& <\left(1+\frac{1}{\Gamma(\gamma+1)}\right)^{-1} .
\end{aligned}
$$

Hence it follows from Theorem 3.2 that (1.1) has at least one nontrivial solution.

In what follows, we study the case where nonlinear term is controlled by power functions, the main tool is the following Schauder fixed point theorem.

Lemma 3.5 ([4, Schauder fixed point theorem]). Let $\mathrm{T}$ be a continuous and compact mapping of a Banach space E into itself, such that the set

$$
\{z \in \mathrm{E}: z=\lambda \mathrm{T} z \text { for some } 0 \leqslant \lambda \leqslant 1\}
$$

is bounded. Then $\mathrm{T}$ has a fixed point.

Theorem 3.6. Assume $\mathrm{f}(\mathrm{t}, 0,0) \not \equiv 0$ for any $\mathrm{t} \in[0,1]$, and there exist three nonnegative functions $\mathrm{p}, \mathrm{q}, \mathrm{r} \in \mathrm{L}^{1}[0,1]$ and nonnegative constants $\epsilon_{1} \neq 1, \epsilon_{2} \neq 1$ such that

$$
\left|f\left(t, z_{1}, z_{2}\right)\right| \leqslant p(t)\left|z_{1}\right|^{\epsilon_{1}}+q(t)\left|z_{2}\right|^{\epsilon_{2}}+r(t) \text {, a.e. }\left(t, z_{1}, z_{2}\right) \in[0,1] \times \mathbb{R}^{2} .
$$

Then (1.1) has at least one nontrivial solution.

Proof. According to Lemma $2.9, \mathrm{~T}: \mathrm{C}[0,1] \rightarrow \mathrm{C}[0,1]$ is completely continuous operator. Take

$$
a=\left(1+\frac{1}{\Gamma \epsilon_{1}(\gamma+1)}\right) \int_{0}^{1} \mathcal{H}(s)[p(s)+q(s)] d s, \quad b=\int_{0}^{1} \mathcal{H}(s) q(s) d s .
$$

Choose

$$
\sigma \geqslant \max \left\{3 b,(3 a)^{\frac{1}{1-\epsilon_{1}}},(3 a)^{\frac{1}{1-\epsilon_{2}}}\right\}
$$

and define a closed ball $\Omega_{\sigma}=\{y \in C[0,1]:\|y\| \leqslant \sigma\}$. For every $y \in \Omega_{\sigma}$, one has

$$
|T y(t)| \leqslant \int_{0}^{1} K(t, s)\left|f\left(s, I^{\gamma} y(s), y(s)\right)\right| d s \leqslant \int_{0}^{1} \mathcal{H}(s)\left|f\left(s, I^{\gamma} y_{0}(s), y_{0}(s)\right)\right| d s
$$


It follows from (3.12) that

$$
\begin{aligned}
\left|f\left(s, I^{\gamma} y(s), y(s)\right)\right| & \leqslant p(s)\left|I^{\gamma} y(s)\right|^{\epsilon_{1}}+q(s)|y(s)|^{\epsilon_{2}}+r(s) \\
& \leqslant \frac{\|y\|^{\epsilon_{1}} p(s)}{\Gamma^{\epsilon_{1}}(\gamma+1)}+\|y\|^{\epsilon_{2}} q(s)+r(s) \\
& \leqslant\left(\|y\|^{\epsilon_{2}}+\frac{\|y\|^{\epsilon_{1}}}{\Gamma^{\epsilon_{1}}(\gamma+1)}\right)[p(s)+q(s)]+r(s) \\
& \leqslant\left(1+\frac{1}{\Gamma^{\epsilon_{1}}(\gamma+1)}\right)\left(\|y\|^{\epsilon_{1}}+\|y\|^{\epsilon_{2}}\right)[p(s)+q(s)]+r(s) .
\end{aligned}
$$

In view of (3.13)-(3.15), we obtain the following estimate:

$$
\begin{aligned}
|\mathrm{Ty}(\mathrm{t})| & \leqslant\left(1+\frac{1}{\Gamma^{\epsilon_{1}}(\gamma+1)}\right) \int_{0}^{1} \mathcal{H}(s)\left(\|y\|^{\epsilon_{1}}+\|y\|^{\epsilon_{2}}\right)[\mathrm{p}(\mathrm{s})+\mathrm{q}(\mathrm{s})] \mathrm{d} s+\int_{0}^{1} \mathcal{H}(\mathrm{s}) \mathrm{r}(\mathrm{s}) \mathrm{d} s \\
& =\mathrm{a}\left(\|y\|^{\epsilon_{1}}+\|y\|^{\epsilon_{2}}\right)+\mathrm{b} \leqslant \mathrm{a} \sigma^{\epsilon_{1}}+a \sigma^{\epsilon_{2}}+\mathrm{b} \leqslant \frac{\sigma}{3}+\frac{\sigma}{3}+\frac{\sigma}{3}=\sigma,
\end{aligned}
$$

which implies that $\|\mathrm{Ty}\| \leqslant \sigma$. Thus we have $\mathrm{T}: \Omega_{\sigma} \rightarrow \Omega_{\sigma}$ and

$$
\left\{y \in \Omega_{\sigma}: y=\lambda T y \text { for some } 0 \leqslant \lambda \leqslant 1\right\}
$$

is bounded. According to the Schauder fixed point theorem, T has a fixed point $y^{*} \in \bar{\Omega}$, since $f(t, 0,0) \not \equiv 0$, by Lemma 2.7, the solution $y^{*}$ of the (2.1) is nontrivial, furthermore, (1.1) has at least a nontrivial solution $z^{*}=\mathrm{I}^{\gamma} \mathrm{y}^{*}$. The proof of Theorem 3.6 is completed.

Remark 3.7. Theorems 3.2 and 3.6 cover all cases $\epsilon_{1}, \epsilon_{2} \in[0, \infty)$, especially, if $\epsilon_{1}=\epsilon_{2}=0, \sigma$ in (3.13) can be chosen as the following way

$$
\sigma \geqslant \max \{3 b, 3 a\} .
$$

Corollary 3.8. Assume $\mathrm{f}(\mathrm{t}, 0,0) \not \equiv 0$ for any $\mathrm{t} \in[0,1]$, and there exist two nonnegative constants $\epsilon_{1} \neq 1, \epsilon_{2} \neq 1$ such that

$$
\lim _{\left|z_{1}\right|+\left|z_{2}\right| \rightarrow \infty} \frac{\left|f\left(t, z_{1}, z_{2}\right)\right|}{\left|z_{1}\right|^{\epsilon_{1}}+\left|z_{2}\right|^{\epsilon_{2}}}<\infty
$$

uniformly holds for $\mathrm{t} \in[0,1]$. Then (1.1) has at least one nontrivial solution.

Proof. In fact, by (3.16), there exists $N>0$ such that for $\left|z_{1}\right|+\left|z_{2}\right|>N$ and $t \in[0,1]$,

$$
\left|f\left(t, z_{1}, z_{2}\right)\right| \leqslant M\left(\left|z_{1}\right|^{\epsilon_{1}}+\left|z_{2}\right|^{\epsilon_{2}}\right) \text {. }
$$

On the other hand, it follows from the continuity of $f$ that there exists $M_{0}>0$ such that for any $\left|z_{1}\right|+\left|z_{2}\right| \leqslant$ $\mathrm{N}$ and $\mathrm{t} \in[0,1]$,

$$
\left|f\left(t, z_{1}, z_{2}\right)\right| \leqslant M_{0}
$$

Thus, we have

$$
\left|\mathbf{f}\left(\mathrm{t}, z_{1}, z_{2}\right)\right| \leqslant M\left(\left|z_{1}\right|^{\epsilon_{1}}+\left|z_{2}\right|^{\epsilon_{2}}\right)+M_{0},\left(t, z_{1}, z_{2}\right) \in[0,1] \times \mathbb{R}^{2} .
$$

According to Theorem 3.6, the conclusion of Corollary 3.8 holds.

Now we focus on the uniqueness of the nontrivial solution for (1.1), we have the following theorem.

Theorem 3.9. Suppose $\mathrm{f}(\mathrm{t}, 0,0) \not \equiv 0$ for any $\mathrm{t} \in[0,1]$ and there exist nonnegative functions $\mathrm{p}, \mathrm{q} \in \mathrm{L}^{1}[0,1]$ such that

$$
\left|f\left(t, z_{1}, z_{2}\right)-f\left(t, w_{1}, w_{2}\right)\right| \leqslant p(t)\left|z_{1}-w_{1}\right|+q(t)\left|z_{2}-w_{2}\right|, \text { a.e. }\left(t, z_{1}, z_{2}\right),\left(t, w_{1}, w_{2}\right) \in[0,1] \times \mathbb{R}^{2} .
$$

Then (1.1) has a unique nontrivial solution if

$$
\int_{0}^{1} \mathcal{H}(\mathrm{s})[\mathrm{p}(\mathrm{s})+\mathrm{q}(\mathrm{s})] \mathrm{d} \mathrm{s}<\left(1+\frac{1}{\Gamma(\gamma+1)}\right)^{-1} .
$$


Proof. Firstly let $w_{1}=w_{2} \equiv 0$, we have

$$
\left|\mathbf{f}\left(\mathrm{t}, z_{1}, z_{2}\right)\right| \leqslant p(t)\left|z_{1}\right|+q(t)\left|z_{2}\right|+|f(t, 0,0)| .
$$

It follows from Theorem 3.2, that there exists a nontrivial solution for (1.1).

But in this case, we prefer to obtain uniqueness of nontrivial solution for (1.1). To do this, we only need to prove the operator T given in (2.10) is a contraction. Similar to (3.6), we have

$$
\left|f\left(s, I^{\gamma} u(s), u(s)\right)-f\left(s, I^{\gamma} v(s), v(s)\right)\right| \leqslant\left(1+\frac{1}{\Gamma(\gamma+1)}\right)[p(s)+q(s)]|| u-v \| .
$$

And then

$$
\begin{aligned}
\|\mathrm{Tu}-\mathrm{T} v\| & \leqslant \int_{0}^{1} \mathcal{H}(\mathrm{s})\left|\mathrm{f}\left(\mathrm{s}, \mathrm{I}^{\gamma} \mathrm{u}(\mathrm{s}), \mathrm{u}(\mathrm{s})\right)-\mathrm{f}\left(\mathrm{s}, \mathrm{I}^{\gamma} v(\mathrm{~s}), v(\mathrm{~s})\right)\right| \mathrm{d} \mathrm{s} \\
& \leqslant\left(1+\frac{1}{\Gamma(\gamma+1)}\right) \int_{0}^{1} \mathcal{H}(\mathrm{s})[\mathrm{p}(\mathrm{s})+\mathrm{q}(\mathrm{s})] \mathrm{d} s\|\mathrm{u}-v\|=\mathrm{m}\|\mathrm{u}-\boldsymbol{v}\|,
\end{aligned}
$$

which implies that $T$ is indeed a contraction since $m<1$. By the Banach fixed point theorem, (1.1) has a unique nontrivial solution.

\section{Numerical examples}

Example 4.1. Consider the existence of nontrivial solutions for the nonlinear fractional differential equation

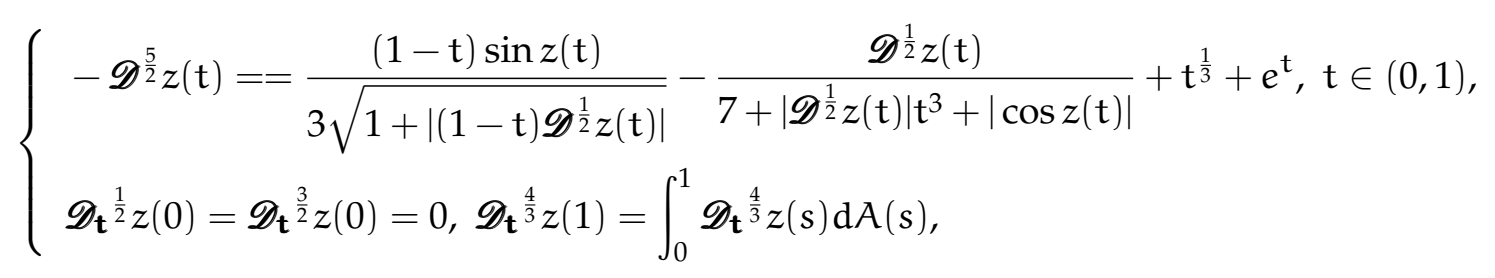

where $A$ is a function of bounded variation

$$
A(t)= \begin{cases}0, & t \in\left[0, \frac{1}{2}\right), \\ \frac{3}{2}, & t \in\left[\frac{1}{2}, \frac{3}{4}\right), \\ 1, & t \in\left[\frac{3}{4}, 1\right] .\end{cases}
$$

Then (4.1) has at least one nontrivial solution.

Proof. From (4.2), by simple computation, (4.1) is equivalent to a 4-point boundary value problem with coefficients of both signs

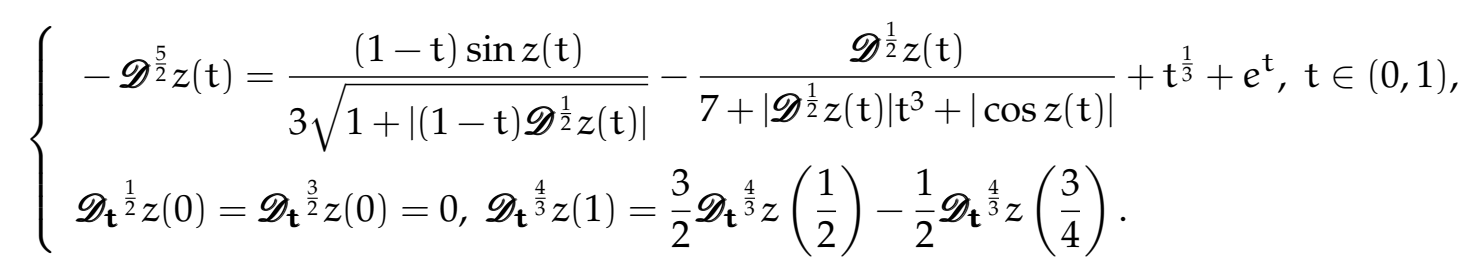

Thus we only need to study the 4-point boundary value problem (4.3).

Let $\alpha=\frac{5}{2}, \gamma=\frac{1}{2}, \mu=\frac{4}{3}$, and set

$$
f\left(t, z_{1}, z_{2}\right)=\frac{(1-t) \sin z_{1}}{3 \sqrt{1+\left|z_{2}\right|}}-\frac{(1-t) z_{2}}{7+\left|z_{3}\right| t^{3}+\left|z_{1}\right|}+t^{\frac{1}{3}}+e^{t}, \quad p(t)=\frac{1-t}{3}, q(t)=\frac{1-t}{7}, r(t)=t^{\frac{1}{3}}+e^{t}
$$

Then

$$
\left|f\left(t, z_{1}, z_{2}\right)\right| \leqslant p(t)\left|z_{1}\right|+q(t)\left|z_{2}\right|+r(t),
$$


and

$$
\left(1+\frac{1}{\Gamma(\gamma+1)}\right)^{-1}=\left(\frac{1}{\Gamma\left(\frac{3}{2}\right)}+1\right)^{-1} \approx 0.4698
$$

On the other hand, we have

$$
\begin{aligned}
G(t, s) & = \begin{cases}t(1-s)^{\frac{1}{6}}-(t-s)=: G_{1}(t, s), & 0 \leqslant s \leqslant t \leqslant 1 \\
t(1-s)^{\frac{1}{6}}=: G_{2}(t, s), & 0 \leqslant t \leqslant s \leqslant 1 .\end{cases} \\
\mathcal{C} & =\int_{0}^{1} \frac{\Gamma(\alpha-\mu)}{\Gamma(\alpha-\gamma)} t^{\alpha-\gamma-1} \mathrm{dA}(\mathrm{t})=\frac{\Gamma\left(\frac{7}{6}\right)}{\Gamma(2)} \int_{0}^{1} \mathrm{tdA}(\mathrm{t})=\frac{3 \Gamma\left(\frac{7}{6}\right)}{8 \Gamma(2)} \approx 0.3479, \\
\mathcal{B} & =\frac{\Gamma(\alpha-\mu)}{(1-\mathcal{C}) \Gamma(\alpha-\gamma)}=\frac{\Gamma\left(\frac{7}{6}\right)}{(1-0.3479) \Gamma(2)} \approx 1.4226,
\end{aligned}
$$

thus we have

$$
\mathcal{H}(s)=\frac{(1-s)^{\alpha-\mu-1}}{\Gamma(\alpha-\gamma)}+\mathcal{B}\left|\mathcal{G}_{\mathrm{A}}(s)\right|=(1-s)^{\frac{1}{6}}+1.4226\left|\mathcal{G}_{\mathrm{A}}(\mathrm{s})\right|,
$$

where

$$
\mathcal{G}_{A}(s)= \begin{cases}\frac{3}{2} G_{1}\left(\frac{1}{2}, s\right)-\frac{1}{2} G_{1}\left(\frac{3}{4}, s\right)=\frac{3}{8}(1-s)^{\frac{1}{6}}+s-\frac{3}{8}, & 0 \leqslant s<\frac{1}{2}, \\ \frac{3}{2} G_{2}\left(\frac{1}{2}, s\right)-\frac{1}{2} G_{1}\left(\frac{3}{4}, s\right)==\frac{3}{8}(1-s)^{\frac{1}{6}}-\frac{1}{2} s+\frac{3}{8}, & \frac{1}{2} \leqslant s<\frac{3}{4}, \\ \frac{3}{2} G_{2}\left(\frac{1}{2}, s\right)-\frac{1}{2} G_{2}\left(\frac{3}{4}, s\right)==\frac{3}{8}(1-s)^{\frac{1}{6},} & \frac{3}{4} \leqslant s \leqslant 1 .\end{cases}
$$

It follows from (4.4) and (4.5) that

$$
\begin{aligned}
\int_{0}^{1} \mathcal{H}(\mathrm{s})(\mathrm{p}(\mathrm{s})+\mathrm{q}(\mathrm{s})) \mathrm{d} s= & \frac{1}{10} \int_{0}^{1}(1-\mathrm{s})^{\frac{5}{6}} \mathrm{~d} s+0.14226 \int_{0}^{1}\left|\mathcal{G}_{\mathrm{A}}(\mathrm{s})\right|(1-\mathrm{s}) \mathrm{d} \mathrm{s} \\
= & \frac{6}{110}+0.14226\left[\left(\frac{9}{44}\left(1-\left(\frac{1}{2}\right)^{\frac{11}{6}}\right)+\frac{1}{12}-\frac{3}{64}\right)\right. \\
& \left.+\left(\frac{9}{44}\left(\left(\frac{1}{2}\right)^{\frac{11}{6}}-\left(\frac{1}{4}\right)^{\frac{11}{6}}\right)-\frac{11}{384}+\frac{9}{256}\right)+\frac{9}{44}\left(\frac{1}{4}\right)^{\frac{11}{6}}\right] \\
\approx & 0.08973<0.4698,
\end{aligned}
$$

which implies that the condition (3.2) in Theorem 3.2 is satisfied, and from Theorem 3.2 the nonlinear fractional differential equation (4.1) has a nontrivial solution.

Example 4.2. Consider the uniqueness of nontrivial solutions of the nonlinear fractional differential equation (4.1) with nonlinear term

$$
f\left(t, z(t), \mathscr{D}_{\mathbf{t}}^{\gamma} z(t)\right)=\frac{(1-t) \sin z(t)}{3}-\frac{\cos \left(\mathscr{D}^{\frac{1}{2}} z(t)-t^{\frac{1}{2}} z(t)\right)}{7}+e^{t},
$$

then the above equation has a unique nontrivial solution.

Proof. In fact, let

$$
f\left(t, z_{1}, z_{2}\right)=\frac{(1-t) \sin z_{1}}{3}-\frac{\cos \left(z_{2}-t z_{2}\right)}{7}+e^{t}
$$

then

where

$$
\left|\mathbf{f}\left(\mathrm{t}, z_{1}, z_{2}\right)-\mathbf{f}\left(\mathrm{t}, w_{1}, w_{2}\right)\right| \leqslant \frac{1-\mathrm{t}}{3}\left|z_{1}-w_{1}\right|+\frac{1-\mathrm{t}}{7}\left|z_{2}-w_{2}\right|, \mathrm{t} \in[0,1],
$$

$$
p(t)=\frac{1-t}{3}, q(t)=\frac{1-t}{7} .
$$

Thus combining (4.4) and (4.6), Theorem 3.9 guarantees that the above boundary value problem has a unique nontrivial solution. 


\section{Acknowledgment}

The authors were supported financially by the National Natural Science Foundation of China 11571296 and 11371221, and the Natural Science Foundation of Shandong Province of China (ZR2014AM009).

\section{References}

[1] A. Azar, S. Vaidyanathan, A. Ouannas, Fractional Order Control and Synchronization of Chaotic Systems, SpringerVerlag, Germany, (2017). 1

[2] Y. Cui, Y. Zou, An existence and uniqueness theorem for a second order nonlinear system with coupled integral boundary value conditions, Appl. Math. Comput., 256 (2015), 438-444. 1

[3] A. Debbouche, V. Antonov, Finite-dimensional diffusion models of heat transfer in fractal mediums involving local fractional derivatives, Nonlinear Studies, 24 (2017), 527-535. 1

[4] K. Deimling, Nonlinear Functional Analysis, Springer-Verlag, Berlin, (1985). 3.1, 3.5

[5] K. Diethelm, A. D. Freed, On the solutions of nonlinear fractional order differential equations used in the modelling of viscoplasticity, Sci. Comput. Chem. Engin. II, 1999 (1999), 7 pages. 1

[6] W. G. Glöckle, T. F. Nonnenmacher, A fractional calculus approach of self-similar protein dynamics, Biophys J., 68 (1995), 46-53. 1

[7] C. S. Goodrich, Existence of a positive solution to a class of fractional differential equations, Appl. Math. Lett., 23 (2010), 1050-1055. 1

[8] W. Jiang, B. Wang, Z. Wang, The Existence of positive solutions for multi-point boundary value problems of fractional differential equations, Phys. Procedia, 25 (2012), 958-964. 1

[9] N. Laskin, Fractals and quantum mechanics, Chaos, 10 (2000), 780-790. 1

[10] N. Laskin, Fractional quantum mechanics, Phys. Rev. E, 62 (2000), 3135-3145.

[11] N. Laskin, Fractional quantum mechanics and Léry path integrals, Phys. Lett. A, 268 (2000), 298-305.

[12] N. Laskin, Fractional Schrödinger equation, Phys. Rev. E, 2002 (2002), 7 pages. 1

[13] L. Liu, F.-L. Sun, X.-U. Zhang, Y.-H. Wu, Bifurcation analysis for a singular differential system with two parameters via to degree theory, Nonlinear Anal. Model. Control, 22 (2017), 31-50. 1

[14] F. Mainardi, Fractional calculus: Some basic problems in continuum and statistical mechanics, Springer, Vienna, (1997). 1

[15] S. McKee, J. A. Cuminato, A novel variant of a product integration method and its relation to discrete fractional calculus, Appl. Numer. Math., 114 (2017), 179-187. 1

[16] K. S. Miller, B. Ross, An Introduction to the Fractional Calculus and Fractional Differential Equations, John Wiley \& Sons, New York, (1993). 2.1, 2.2, 2.4

[17] I. Podlubny, Fractional Differential Equations, Mathematics in Science and Engineering, Academic Press, New York, (1999). 2.1, 2.2, 2.4

[18] T. Ren, S. Li, X.-G. Zhang, Positive solutions of a weakly singular periodic eco-economic system with changing-sign perturbation, J. Nonlinear Sci. Appl., 10 (2017), 2539-2549. 1

[19] T. Ren, S. Li, X.-G. Zhang, L. Liu, Maximum and minimum solutions for a nonlocal p-Laplacian fractional differentialsystem from eco-economical processes, Bound. Value Probl., 2017 (2017), 15 pages.

[20] F.-L. Sun, L. Liu, X.-G. Zhang, Y.-H. Wu, Spectral analysis for a singular differential system with integral boundary conditions, Mediterr. J. Math., 13 (2016), 4763-4782. 1

[21] J. R. L. Webb, G. Infante, Non-local boundary value problems of arbitrary order, J. Lond. Math. Soc., 79 (2009), $238-258$. 2

[22] X.-J. Yang, Fractional derivatives of constant and variable orders applied to anomalous relaxation models in heat-transfer problems, Therm. Sci., 21 (2017), 1161-1171. 1

[23] X.-J. Yang, J. A. Machado, D. Baleanu, Anomalous diffusion models with general fractional derivatives within the kernels of the extended Mittag-Leffler type functions, Rom. Rep. Phys., 2017 (2017), 19 pages. 1

[24] X.-J. Yang, H. M. Srivastava, J. A. Tenreiro Machadod, A new fractional derivative without singular kernel: Application to the modelling of the steady heat flow, Therm. Sci., 20 (2017), 753-756; 1

[25] X.-G. Zhang, Y.-F. Han, Existence and uniqueness of positive solutions for higher order nonlocal fractional differential equations, Appl. Math. Lett., 25 (2012), 555-560. 1

[26] X.-G. Zhang, L. Liu, B. Wiwatanapataphee, Y.-H. Wu, Positive solutions of eigenvalue problems for a class of fractional differential equations with derivatives, Abstr. Appl. Anal., 2012 2012, 16 pages.

[27] X.-G. Zhang, L. Liu, B. Wiwatanapataphee, Y.-H. Wu, The eigenvalue for a class of singular p-Laplacian fractional differential equations involving the Riemann-Stieltjes integral boundary condition, Appl. Math. Comput., 235 (2014), $412-422$.

[28] X.-G. Zhang, L. Liu, Y.-H. Wu, Existence results for multiple positive solutions of nonlinear higher order perturbed fractional differential equations with derivatives, Appl. Math. Comput., 219 (2012), 1420-1433.

[29] X.-G. Zhang, L. Liu, Y.-H. Wu, The eigenvalue problem for a singular higher fractional differential equation involving fractional derivatives, Appl. Math. Comput., 218 (2012), 8526-8536. 
[30] X.-G. Zhang, L. Liu, Y.-H. Wu, The uniqueness of positive solution for a singular fractional differential system involving derivatives, Commun. Nonlinear Sci. Numer. Simul., 18 (2013), 1400-1409. 1

[31] X.-G. Zhang, L. Liu, Y.-H. Wu, The uniqueness of positive solution for a fractional order model of turbulent flow in a porous medium, Appl. Math. Lett., 37 (2014), 26-33. 1

[32] X.-G. Zhang, L. Liu, Y.-H. Wu, Variational structure and multiple solutions for a fractional advection-dispersion equation, Comput. Math. Appl., 68 (2014), 1794-1805. 1

[33] X.-G. Zhang, L. Liu, Y.-H. Wu, B. Wiwatanapataphee, The spectral analysis for a singular fractional differential equation with a signed measure, Appl. Math. Comput., 257 (2015), 252-263. 1

[34] X.-G. Zhang, L. Liu, Y.-H. Wu, B. Wiwatanapataphee, Nontrivial solutions for a fractional advection dispersion equation in anomalous diffusion, Appl. Math. Lett., 66 (2017), 1-8.

[35] X.-G. Zhang, C. Mao, L. Liu, Y.-H. Wu, Exact iterative solution for an abstract fractional dynamic system model for Bioprocess, Qual. Theory Dyn. Syst., 16 (2017), 205-222.

[36] X.-G. Zhang, Y.-H. Wu, L. Caccetta, Nonlocal fractional order differential equations with changing-sign singular perturbation, Appl. Math. Model., 39 (2015), 6543-6552. 1 